\title{
Genes derived from ancient polyploidy have higher genetic diversity and are associated with domestication in Brassica rapa
}

Xinshuai $\mathrm{Qi}^{1,5}$, Hong An ${ }^{2,5}$, Tara E. Hall ${ }^{1}$, Chenlu Di ${ }^{1}$, Paul D. Blischak ${ }^{1}$, Michael T. W. McKibben $^{1}$, Yue Hao ${ }^{3}$, Gavin C. Conant ${ }^{3,4}$, J. Chris Pires ${ }^{2}$, and Michael S. Barker ${ }^{1}$

1. Department of Ecology \& Evolutionary Biology, University of Arizona, Tucson, Arizona, USA

2. Division of Biological Sciences, University of Missouri, Columbia, Missouri, USA

3. Bioinformatics Research Center, North Carolina State University, Raleigh, North Carolina, USA

4. Department of Biological Sciences, North Carolina State University, Raleigh, North Carolina, USA

5. These authors contributed equally to this work

Correspondence should be addressed to M. S. B. (msbarker@arizona.edu)

\section{Summary}

- Many crops are polyploid or have a polyploid ancestry. Recent phylogenetic analyses have found that polyploidy often preceded the domestication of crop plants. One explanation for this observation is that increased genetic diversity following polyploidy may have been important during the strong artificial selection that occurs during domestication.

- To test the connection between domestication and polyploidy, we identified and examined candidate genes associated with the domestication of the diverse crop varieties of Brassica rapa. Like all "diploid" flowering plants, B. rapa has a diploidized paleopolyploid genome and experienced many rounds of whole genome duplication (WGD). We analyzed transcriptome data of more than 100 cultivated B. rapa accessions. 
- Using a combination of approaches, we identified more than 3,000 candidate genes associated with the domestication of four major B. rapa crop varieties. Consistent with our expectation, we found that the candidate genes were significantly enriched with genes derived from the Brassiceae mesohexaploidy. We also observed that paleologs were significantly more diverse than non-paleologs.

- Our analyses find evidence for that genetic diversity derived from ancient polyploidy played a key role in the domestication of $B$. rapa and provide support for its importance in the success of modern agriculture.

Keywords: Brassica, domestication, polyploidy, whole genome duplication, genomics, genetic diversity

\section{Introduction}

Polyploidy, or whole genome duplication (WGD), has long been associated with crop domestication and diversity (Anderson, 1969; Lewis, 1980; Heiser, 1990; Hilu, 1993; Paterson, 2005; Udall \& Wendel, 2006; Meyer et al., 2012; Renny-Byfield \& Wendel, 2014). Many desirable crop traits such as larger seed size, greater stress tolerance, and increased disease resistance are often attributed to polyploidy (Lewis, 1980; Levin, 1983). A recent phylogenetic analysis found that domesticated plants have experienced significantly more polyploidy than their wild relatives (Salman-Minkov et al., 2016). Polyploidy often precedes domestication and crops are nearly twice as likely to be domesticated in lineages with a relatively recent WGD compared to those without (Salman-Minkov et al., 2016). Among the potential explanations for the relationship between polyploidy and domestication, the expanded genetic diversity and plasticity of polyploid plants may be especially advantageous during domestication and crop improvement (Otto \& Whitton, 2000; Shimizu-Inatsugi et al., 2017; Baduel et al., 2018; Paape et al., 2018; Monnahan et al., 2019). Analyses 
in yeast have shown that polyploid lineages not only have higher genetic diversity but also adapt to new environments faster than their lower ploidal level relatives (Selmecki et al., 2015). Similarly, the niches of polyploid plants evolve faster than their diploid relatives (Baniaga et al., 2020). These features may collectively give polyploids unique advantages over diploids during domestication and the global spread of crops that occurred with human population expansion.

Although nearly $30 \%$ of plant species are recent polyploids, all flowering plants are paleopolyploids with varying histories of WGD (One Thousand Plant Transcriptomes Initiative, 2019). Given that the genetic consequences of polyploidy play out over time as genomes diploidize and paralogs fractionate (Otto, 2007; Arrigo \& Barker, 2012; Baduel et al., 2018), we may expect that the effects of polyploidy extend to diploidized species. Here, we sought to test whether past polyploidy is associated with increased diversity and domestication in the crop varieties of Brassica rapa. Like all flowering plants, the genome of $B$. rapa has been multiplied and fractionated many times over. The most recent polyploidization in the ancestry of $B$. rapa was a mesohexaploidy that occurred approximately 9-28 MYA(Lukens et al., 2004; Lysak et al., 2005; Beilstein et al., 2010; Wang et al., 2011; Arias et al., 2014; Cheng et al., 2014; Franzke et al., 2016). Further, B. rapa has been domesticated into many different crop varieties across Europe and Asia. These include turnips, oil seeds, pak choi, Chinese cabbage, and mustard seeds. Many researchers have suggested that there is a connection between the mesohexaploidy and the diversity of $B$. rapa crop varieties (Cheng et al., 2014, 2016), but the relationship has never been explicitly tested. Using recently sequenced transcriptomes from a diverse array of $B$. rapa accessions (Qi et al., 2017), we tested if polyploid-derived regions of the genome are enriched with candidate genes associated with domestication. We also compared genetic variation in the polyploid vs non-polyploid derived regions of the $B$. rapa genome. Given the frequency of ancient polyploidy and its contribution to the evolution of plants, our analyses demonstrate the key role of polyploidy in the domestication of B. rapa and provide support for its importance in the success of modern agriculture. 


\section{MATERIALS \& METHODS}

Data Sources. RNA-seq data for this study were previously generated by our group (Qi et al., 2017). Based on previous population genomics analyses (Qi et al 2017), a total of 102 B. rapa accessions (Supplementary Table 2) were selected from the USDA GRIN database (http://www.ars-grin.gov/) or from the author's collection to represent the five major $B$. rapa genetic groups. The five genetic groups include an earlier derived Europe and Central Asia group, represented by turnip (TN, B. rapa and B. rapa subsp. rapa, 22 accessions); four derived $B$. rapa groups, represented by Pak choi (PC, B. rapa subsp. chinensis, 25 accessions), Chinese cabbage (CC, B. rapa subsp. pekinensis, 28 accessions), Indian sarson (IS, B. rapa subsp. trilocularis and B. rapa subsp. dichotoma; 20 accessions) and toria (TO, 7 accessions). The last four genetic groups diverged from the TN group about 2400-4100 years ago after the initial B. rapa domestication in European-Central Asia (Qi et al., 2017). Two B. oleracea accessions (SRR630924 and SRR1032050) from the NCBI Sequence Read Archive (SRA) were used as outgroups for analyses.

RNA-seq Variant Calling. Raw reads were cleaned using Trimmomatic version 0.32 (Bolger et al., 2014), and mapped to the reference B. rapa genome (version 1.5, http://brassicadb.org/brad/) with Tophat version 2.0.14 (Trapnell et al., 2009). SNP calling was performed using Samtools version 0.1.18 and bcftools version 0.1.17 (Li et al., 2009; Li, 2011). The resulting VCF files were filtered with the vcfutils.pl script and vcffilter. Only SNPs with depth greater than 10 and variant quality (QUAL) greater than 30 were retained, which included 1.32 million SNPs. Details about sequencing and variant calling were described previously (Qi et al., 2017).

\section{Identifying Genes Derived from the Mesohexaploidy Event (Paleologs). We used} two different approaches to identify paleologs-genes derived from an ancient polyploid event-retained from the Brassiceae mesohexaploidy, the most recent 
polyploidy in the ancestry of $B$. rapa. Both approaches aim to distinguish genes that were born from the mesohexaploidy from genes derived from other types of duplication events, such as tandem, segmental, and other small-scale duplications. Genes that we identify as derived from the Brassiceae mesohexaploidy were labelled as paleologs, and other genes were labelled as non-paleologs. The first approach used synteny and gene age distribution data from only $B$. rapa to infer paleologs. To estimate the age distribution of gene duplications (also called a Ks plot) for $B$. rapa we obtained CDS sequences from the Brassica database (http://brassicadb.org/brad/). The synonymous substitution rate (Ks) of each gene duplication was calculated using DupPipe (Barker et al., 2008, 2010). The gene age distribution was visualized in $\mathrm{R}$ using histograms. The boundaries of the hexaploid peak were determined using EMMIX (McLachlan et al., 1999) by fitting a mixture model of normal distributions to the Ks data (Tiley et al., 2018). One hundred random starting points and $10 \mathrm{k}$-means starting points were used to identify the number of normal distributions (from 1 to 10). The best fit model was selected based on the Bayesian information criterion (BIC) value. Ks nodes with $>50 \%$ likelihood assignment to the mesohexaploidy peak were considered as gene pairs derived from the Brassica hexaploidy event. We combined these age based inferences of paleologs with syntenic data for $B$. rapa. Syntenic gene sets were generated by CoGe SynMap (genomevolution.org/CoGe/SynMap.pl) with a 2: 2 quota-align ratio and default parameters (Tang et al., 2011) using the unmasked $B$. rapa genome (Version 1.5). These two paleolog lists were integrated with an independently estimated $B$. rapa paleolog list (Cheng et al., 2012). Genes present in at least two of these three lists were extracted using the Venn online tool (http://bioinformatics.psb.ugent.be/webtools/Venn/) and were considered to be high confidence paleologs. Genes only present in one of these three lists were considered low confidence paleologs and were not used in our study. Genes not present in any of the above three paleologs lists were considered non-paleologs.

We also developed a second set of paleologs and non-palelogs from a recent multi-genome comparative analysis with POInT, the Polyploidy Orthology Inference 
Tool (Hao et al., 2020). The POInT analysis modeled the history of genome evolution following the Brassiceae mesohexaploidy by comparing the genomes of Arabidopsis thaliana, Brassica oleracea, Brassica rapa, Crambe hispanica, and Sinapis alba (REF). Briefly, the POInT analysis compared each of the Brassiceae species genomes to $A$. thaliana to identify blocks of triple conserved synteny across each of the four genomes. Blocks from each genome were then merged into "pillars" that contain one to three surviving paralogs in each species from the mesohexaploidy. POInT uses a probabilistic approach to assess if a gene belongs to a paleolog pillar. We filtered for genes in the $B$. rapa genome that had a $>95 \%$ probability of assignment to one of these mesohexaploid pillars from the Hao et al. (2020) analysis. These data were used to develop a list of $B$. rapa paleologs that were retained in single, double, or triple copy from the ancient hexaploidy. All other genes in B. rapa were then classified as non-paleologs.

Selection analyses. We used two different methods to identify genes with signals of positive selection in the four derived $B$. rapa genetic groups. Genomic regions with evidence consistent with selective sweeps were detected using SweeD 3.0 (Pavlidis et al., 2013) based on the composite likelihood ratio (CLR) test of SNP site frequency spectrum (SFS) patterns. A total of 1.32 million SNPs were used in the SweeD tests for the TN group and each of the four derived $B$. rapa genetic groups. These analyses were performed with the default settings except each chromosome was divided to 60,000 grids. The CLR was calculated for each equally generated relative position on each chromosome. Only the top $1 \%$ significant outlier regions were considered. Genes within the outlier regions were then annotated based on B. rapa genome v1.5.

We also used the McDonald-Kreitman test ( $M-K$ test) (McDonald \& Kreitman, 1991) to identify genes experiencing positive selection. This method is based on the proportion of synonymous and nonsynonymous substitutions within and between species that are due to directional selection. For the M-K test, we compared SNPs specific to each of the four $B$. rapa crop groups with the two B. oleracea outgroup 
accessions. For each separate SNP dataset, we annotated synonymous and nonsynonymous SNPs using SnpEff v4.2 (Cingolani et al., 2012). The reference genome database was created using $B$. rapa genome $v 1.5$. The significance of the $M-K$ test was evaluated using Fisher's exact test.

Differential gene expression analysis. Differential gene expression analysis was performed following the Tuxedo protocol (Trapnell et al., 2012). Briefly, cleaned reads were first mapped to the B. rapa genome with TopHat (Trapnell et al., 2009), then mapped BAM files were assembled in Cufflinks (Trapnell et al., 2010), merged with Cuffmerge, quantified in Cuffdiff (Trapnell et al., 2013) (Cufflinks version2.2.1) with multiple-testing corrected q-values as 0.05, and finally indexed and visualized in CummeRbund version 2.14 (Goff et al., 2012). Genes significantly differentially expressed between the TN group and the four derived $B$. rapa groups were identified using the getSig function in CummeRbund (Goff et al., 2012).

Candidate genes from the literature. To confirm our findings, we compared our observations of paleolog enrichment with a list of candidate genes from published studies of $B$. rapa. We surveyed the literature for fine mapping and bulk segregant analyses of $B$. rapa that mapped traits to one or a few candidate genes. These studies and the candidate genes are listed in Supplemental Table 7. Overall, our survey identified 40 candidate genes in $B$. rapa that have been mapped to the location of classical loci associated with crop traits.

Statistical analyses. To test for evidence of paleolog enrichment in our candidate gene lists, we used $2 \times 2$ chi-square tests. We compared the numbers of paleologs and non-paleologs that were not candidate genes to the numbers of paleolog and non-paleolog candidate genes. For each candidate gene list, we aggregated the candidate genes of the four derived $B$. rapa genetic groups. The ratio of paleologs and non-paleologs among non-candidate genes in the $B$. rapa genome was used as the 
expected value for the chi-square test to determine if the candidate gene lists contained more paleologs than expected.

To demonstrate the logical relations among these lists, Venn diagrams were generated using the online Venn diagram tool

(http://bioinformatics.psb.ugent.be/webtools/Venn/). The density distribution of the identified paleologs, non-paleologs, SweeD outlier gene, M-K test outlier gene and differentially expressed gene were visualized in Circos with a bin size of $100 \mathrm{kbp}$. We used the dhyper function in $\mathrm{R}$ to test for significant overlap among the different candidate gene lists.

Nucleotide diversity of $\boldsymbol{B}$. rapa genes. The nucleotide diversity $(\pi)$ of each paleolog and non-paleolog gene was estimated using a custom script from the output of VCFtools (Danecek et al., 2011). The results were summarized and visualized in $\mathrm{R}$ v3.6.1 (R Core Team, 2019).

As a secondary verification of our calculations for nucleotide diversity in paleologs versus non-paleologs, we remapped the filtered RNAseq reads to the $B$. rapa reference genome using STAR v2.7.3a (Dobin et al., 2013). We then used the resulting BAM files to calculate nucleotide diversity using genotype likelihoods with ANGSD v0.921 (Korneliussen et al., 2014). This procedure was done twice, once filtering for uniquely mapping reads and once without, to see if the results differed based on potential read mismapping caused by gene duplicates. For these calculations, we first inferred the folded site frequency spectrum (-doSaf 1, -fold 1) and used this as a point estimate for estimating theta (-doThetas 1) at each site separately for all paleologs and nonpaleologs (Nielsen et al., 2012; Korneliussen et al., 2013). We then used the thetaStat program within ANGSD to calculate nucleotide diversity for each gene. We also compared the distributions of nucleotide diversity between paleologs and nonpaleologs using both t-tests and non-parametric Mann-Whitney U-tests within R v3.6.1 (R Core Team, 2019). 


\section{Results}

\section{Partitioning the $B$. rapa genome into paleologs vs non-paleologs}

To test the contribution of paleopolyploidy to the domestication of Brassica rapa, we used two different approaches to classify genes as paleologs-genes derived from the Brassica mesohexaploidy-or non-paleologs. In the first approach, we used gene age distributions and synteny in B. rapa. An initial list of putative paleologs was generated from pairs of genes with synonymous divergence (Ks) values that correspond to the peak of duplication associated with the Brassiceae mesohexaploidy. This approach identified 21,280 genes that are likely derived from the mesohexaploidy (Fig. 1a). This result is consistent with previous Ks estimates (Barker et al., 2009;

Cheng et al., 2013). Ancient WGDs and their associated paralogs may also be classified by identifying syntenic blocks of duplication. Syntenic analyses with CoGe recovered 19,810 syntenic gene sets that contained 31,796 paleologs. Finally, we compared these two lists of putative paleologs with a previously curated list of 23,716 paleologs reported by an independent research group (Cheng et al., 2012). Genes that appeared at least twice in these three lists were classified as paleologs and were used in further analyses (Fig. 1b). The final paleolog list included 27,919 genes (Supplementary Table 1), which represents $68.06 \%$ of the 41,020 annotated genes in the $B$. rapa reference genome (Version 1.5). We also considered the 5,424 genes that were not present in any of the above three paleolog lists as non-paleologs (Fig. 1b), which represents $13.22 \%$ of the total $B$. rapa genes. Paleologs and non-paleologs are distributed throughout the $B$. rapa genome (Fig. 2).

We also used results from a recent multi-genome comparative analysis (Hao et al., 2020) to infer paleologs in B. rapa. A recent POInT analysis of the genomes for four Brassiceae species (Brassica oleracea, Brassica rapa, Crambe hispanica, and Sinapis alba) uncovered evidence for 14,050 regions ("pillars" in POInT terminology) of triple conserved synteny from the Brassiceae mesohexaploidy across the four genomes. Genes in these regions of conserved synteny are present in one to three copies in each genome. After parsing these output for $B$. rapa genes that were assigned to these 
regions with $>95 \%$ probability by POInT, we identified 4,818 paleologs retained as single copy genes, 6,128 paleologs retained in duplicate, and 2,553 retained in triplicate (FIG 1b; Supplementary Table 1). The remaining 27,527 genes in the B. rapa genome were considered non-paleologs. Using this approach, only $32.9 \%$ of the $B$. rapa genome were identified as paleologs, a much lower fraction than the above synteny + Ks $B$. rapa only method. Despite the difference in the number of inferred paleologs, there was a high overlap in the lists with $89.2 \%$ of the paleologs inferred by POInT also found by the $B$. rapa only approach. Most of the difference in the overall number is likely due to the POInT approach's initial filtering for regions of the genome that have been retained in triplicate and requiring that each of these regions is found across all species, greatly reducing the number of paleologs identified. In contrast, the B. rapa only approach recovers more paleologs because it only requires regions to be retained in duplicate in the genome of only one species (B. rapa). Notably, a less stringent version of the POInT results (not presented) that included all possible identified paleologs regardless of assignment probability identified 22,774 paleologs with $89.5 \%$ overlap with the $B$. rapa only list. Thus, these methods appear to be largely identifying the same genes as paleologs in the $B$. rapa genome. These methods provide two different assessments of paleolog identity that we used to further explore the relationship between polyploidy and domestication of $B$. rapa.

\section{Analyses of selection identify candidate genes associated with domestication of $B$. rapa crop varieties}

We used a diverse collection of sequenced transcriptome data from across five groups to identify candidate genes associated with the domestication and improvement of $B$. rapa crop varieties. In total, 1.32 million SNPs from transcriptome data with 25X coverage or greater from 102 B. rapa accessions were analyzed (Supplementary Table 2). Based on our previous population genomic analyses of these data (Qi et al., 2017), the accessions are comprised of a European-Central Asian $B$. rapa population represented by turnip (TN, B. rapa subsp. rapa) and four derived 
groups represented by pak choi (PC, B. rapa subsp. chinensis), Chinese cabbage (CC, $B$. rapa subsp. pekinensis), Indian sarson (IS, B. rapa subsp. trilocularis) and toria (TO, $B$. rapa subsp. dichotoma). We analyzed these data with an ensemble of molecular evolution and population genomic approaches to identify candidate genes associated with each group.

During domestication and crop improvement, we expect genetic variation important for agricultural traits to be selected in crop populations. To identify genes or genomic regions that have experienced recent positive selection, we used two different approaches. First, we used a selective sweep test, SweeD (Pavlidis et al., 2013), to identify regions associated with significantly reduced variation consistent with a recent selective sweep in each of the crop varieties. We identified 3,387 unique genes within the identified selective sweep regions (Fig. 3a, Supplementary Tables 3 and 4, and Supplementary Fig. 1 and 2). This included 1,072 genes in chinensis, 687 genes in pekinensis, 1,048 genes in toria, 1,228 genes in the sarsons, and 1,713 genes in European-Central Asian group. On average 70\% of these genes were found in only one crop variety (Fig. 3a), indicating that many of these genes may have swept during the putative independent domestication of each crop variety. We also used the McDonald-Kreitman test (M-K test) (McDonald \& Kreitman, 1991) to identify coding regions with a significant excess of fixed amino acid substitutions, a different signature of positive selection. We used B. oleracea as an outgroup. Analyzing each crop variety with the $\mathrm{M}-\mathrm{K}$ test, we found 92 genes in total with a molecular evolutionary signature of positive selection (Fig. 3b and Supplementary Tables 3 and 4). All but one of these genes was uniquely identified in a different crop variety, similar to the selective sweep analysis and consistent with independent domestication and differentiation of these $B$. rapa crops. Overall, we identified 3,479 candidate genes that may be associated with the domestication and improvement of the five $B$. rapa crop groups.

We may also expect significant changes in gene expression to occur during domestication and crop improvement that may not be apparent in analyses of positive selection. To identify candidate genes with changes in gene expression during 
domestication, we performed differential gene expression (DGE) analyses with the four derived B. rapa genetic groups. RNA-seq data for each individual was collected 20 days after germination and the expression levels of genes in each group were compared to gene expression levels in the TN group. We identified a total of 7,813 genes with significant differential expression when comparing each group with the TN population (Fig. 3c, Supplementary Tables 3 and 4, and Supplementary Fig. 2 and 3). Unlike the analyses of selection above, the number of unique differentially expressed genes varied widely among the different crop varieties. The sarsons (IS) were the most differentiated from TN and the other crops with over $61 \%$ of their differentially expressed genes unique. The sarsons also had the largest number of differentially expressed genes at 5,804. In contrast, pak choi (PC) had the smallest number of differentially expressed genes, 1,301, and the lowest percentage that were uniquely different at $14.2 \%$. Other crop varieties fell between these values. The genes identified by all three methods were largely different among all the crops (Fig. 3d) with only a small number of genes identified by one or more of these approaches (i.e., sweep test, M-K test, or DGE analyses). However, the number of overlapping genes found by each of these approaches was significant in all cases based on the expected numbers from a hypergeometric distribution (MK:SweeD overlap $p=0.0013$; MK:DGE overlap $p<$ 0.0001; SweeD:DGE overlap $p<0.0001$ ). This suggests that we are detecting shared signatures of domestication across these different candidate gene approaches. Similarly, most of the genes found to be associated with recent positive selection or differential gene expression were unique to a particular crop lineage as expected with independent domestication and differentiation of these distinct crop varieties.

Across all of the analyses, only five genes were repeatedly present in tests among one or more of the crop varieties (Fig. 3d and Supplementary Table 5). These genes-Bra010933, Bra023190, Bra031452, Bra003055, and Bra035693-were identified in some combination of the three inference methods with evidence for both signatures of positive selection and changes in gene expression. Considering that we recovered these five genes using different approaches, they may play an important role 
during the differentiation and improvement of $B$. rapa crop varieties. Searches of these genes and their Arabidopsis homologs in the STRING (Szklarczyk et al., 2019) and UniProt (UniProt Consortium, 2018) databases recovered a diverse range of potential functions. Bra023190 is uncharacterized in B. rapa, but is homologous with SGR2 in Arabidopsis thaliana, a gene associated with negative gravitropism and leaf movement in darkness (Kato et al., 2002; Mano et al., 2006). Notably, this gene was identified as a candidate gene in both of the cabbage crops analyzed here, $B$. rapa chinensis and pekinensis. Other genes are homologs with $A$. thaliana genes implicated in responses to oxidative stress from photooxidation (Bra010933), salt stress (Bra031452; (Tuteja et al., 2012)), and a vacuolar V-type proton ATPase (Bra035693). Finally, Bra003055, identified by all three candidate gene approaches in the sarsons, was previously found to be over-expressed in B. rapa in soils that are deficient in iron and with an excess of zinc (Li et al., 2014).

\section{Paleologs from the Brassica mesohexaploidy and domestication}

To test if the candidate genes associated with domestication of the B. rapa crop varieties are enriched with paleologs, we compared the number of paleologs in the candidate gene lists to the expected number from our two genome-wide surveys (Fig. 1). We found that the candidate genes were significantly enriched with paleologs from the Brassiceae mesohexaploidy using either paleolog inference method (Fig. 4). Using paleologs inferred with the B. rapa only synteny + Ks approach (Fig. 1a), genes from all three candidate gene approaches were enriched across all crops with paleologs comprising 78.54\% of SweeD $\left(\chi^{2}=160.06, p<0.001\right)$, 83.7\% of McDonald Kreitman $\left(\chi^{2}=10.33, p=0.0013\right)$, and $78.6 \%$ of differentially expressed candidate genes $\left(\chi^{2}=\right.$ $345.40, p<0.001$ ). Similarly, we found that candidate genes were significantly enriched with paleologs inferred with the POInT multi-genome approach (Fig. 1b). Using the POInT defined paleologs, we found that $42.6 \%$ of SweeD $\left(\chi^{2}=157.84, p<\right.$ $0.001), 53.2 \%$ of McDonald Kreitman $\left(\chi^{2}=16.40, p<0.001\right)$, and $40.3 \%$ of the 
differentially expressed candidate genes $\left(\chi^{2}=239.04, p<0.001\right)$ were paleologs. The POInT method allowed us to identify genes retained in single, double, and triple copy from the Brassiceae mesohexaploidy and we found that these varied in their enrichment among the candidate gene lists (Fig. 4; Supplementary Table 6). Paleologs that have returned to single copy in $B$. rapa were significantly enriched in all of the candidate gene lists, whereas paleologs that are retained in duplicate were enriched in the SweeD and differentially expressed gene lists. In contrast, paleologs that have retained all of their copies since the mesohexaploidy-those that are retained in triplicate-were only found to be significantly enriched in the differentially expressed gene candidate gene list, but not in either candidate gene list where there is evidence of significant protein coding changes among the genes. Overall, these results suggest that genes derived from the Brassiceae mesohexaploidy were preferentially selected during the domestication of the $B$. rapa crops.

To further test if domestication genes in B. rapa are significantly enriched with paleologs, we also developed a list of candidate genes from the literature. We focused on studies published over the last 10 years that identified genes through other approaches, such as fine mapping or bulk segregant analysis, to better establish a causal relationship between loci and crop traits. In total, we identified 40 candidate genes that fit these criteria from the literature (Supplementary Table 5). Many of these genes are associated with leaf and seed color variation, clubroot resistance, and cuticular wax biosynthesis. Notably, 15 of these genes were recovered in our candidate gene scans (Supplementary Table 7). Four of these genes were identified in our selective sweep and differential gene expression analyses. Mapping studies previously identified these genes as being associated with leaf color variation (Bra006208)(Fu et al., 2019), cuticular wax biosynthesis (Bra011470 and Bra032670; (Wang et al., 2017, 2019), and clubroot resistance (Bra019410; (Yu et al., 2016). For other loci, mapping studies have identified a small collection of candidate genes in target regions. For example, Rcr5 is a gene of major effect for clubroot resistance in Brassica rapa. A recent bulk segregant analysis and fine mapping study found eight genes in the Rcr5 
target region of $B$. rapa (Huang et al., 2019). In our analyses, three genes in the center of their region were found to be significantly differentially expressed. These results suggest that future mapping studies in B. rapa may be able to leverage our candidate gene lists to improve gene identification.

How many of the candidate genes from our literature survey were paleologs? Of the 40 genes identified in the literature, 36 were paleologs with the $B$. rapa synteny + Ks method and 18 were paleologs with the POInT approach (Fig. 4). The literature candidate genes were significantly enriched with paleologs defined by the $B$. rapa only approach $\left(\chi^{2}=8.85, p=0.0029\right)$ similar to our three different candidate gene lists. Although the percentage of POInT paleologs was higher in the literature candidate gene list than the rest of the genome (Fig. 4), it was not a significant difference $\left(\chi^{2}=\right.$ 2.13, $p=0.1441)$. Given these literature-based candidate genes were largely identified with mapping based approaches rather than genomic scans, these results suggest that the paleolog enrichment observed in our analyses is not likely an artifact of the inference methods. Overall, our results indicate that paleologs were an important source of variation for domestication in B. rapa.

Why did artificial selection preferentially target paleologs? One possible explanation is that these genes may harbor more genetic diversity due to their paralogous history over the past 20 million years. To test this hypothesis, we used three different approaches to examine the nucleotide diversity per gene across the $B$. rapa genome. First, we estimated nucleotide diversity in VCFtools using only uniquely mapped reads from Tophat to minimize error from incorrectly mapped reads. We found that the genes derived from the mesohexaploidy were significantly more diverse than the other genes in the genome regardless of how we identified paleologs (Fig. 5). Paleologs identified by the $B$. rapa only approach were significantly more diverse than non-paleologs $\left(t=43.721, \mathrm{df}=6535, p<2.2 \times 10^{-16}\right.$ for $\mathrm{t}$-test; $\mathrm{W}=70,733,526$ and $p<$ $2.2 \times 10^{-16}$ for $U$-test), and had four times the mean and an order of magnitude more median nucleotide diversity (mean $\pi=0.408 \times 10^{-3}$, median $\pi=0.170 \times 10^{-3}$ ) than the non-paleolog fraction of the genome (mean $\pi=0.111 \times 10^{-3}, \pi=0.124 \times 10^{-4}$ ). 
This was consistent when considering only synonymous or non-synonymous substitutions (Fig. S4). POInT paleologs were also significantly more diverse than non-paleologs $\left(t=14.828, \mathrm{df}=25101, p<2.2 \times 10^{-16}\right.$ for $\mathrm{t}$-test; $\mathrm{W}=128,146,882$ and $p<2.2 \times 10^{-16}$ for $U$-test), with a more modest difference in mean paleolog nucleotide diversity (mean $\pi=0.433 \times 10^{-3}$, median $\pi=0.217 \times 10^{-3}$ ) compared to non-paleologs (mean $\pi=0.335 \times 10^{-3}$, median $\pi=0.713 \times 10^{-4}$ ). However, the median nucleotide diversity of POInT paleologs was still an order of magnitude higher than non-paleologs. All categories of retained POInT paleologs-single, double, and triple retained copies-were significantly more diverse than the non-paleologs (Supplemental Table 6).

To further validate these results, we re-mapped reads with STAR (Dobin et al., 2013) and calculated nucleotide diversity with ANGSD (Korneliussen et al., 2014). Although the empirical estimates of $\pi$ were different because of differences in how $\pi$ is calculated in these approaches, the ANGSD results recapitulated those from Tophat and VCFtools (Fig. S5). Focussing on the paleologs inferred with the B. rapa synteny + Ks approach, paleologs were again found to have significantly higher levels of diversity than non-paleologs (Fig. S4; $t=48.997$, df $=25842, p<2.2 \times 10^{-16}$ for t-test; $W=$ $238,210,946$ and $p<2.2 \times 10^{-16}$ for U-test). Importantly, these results do not differ when filtering for only uniquely mapping reads $(t=44.11$, $d f=26044$, and $p<2.2 x$ $10^{-16}$ for t-test; $W=232,582,598$ and $p<2.2 \times 10^{-16}$ for $U$-test), demonstrating that our results are unlikely to be affected by read mismapping. The higher genetic diversity of paleologs in $B$. rapa may have been important for the rapid response of these plants to artificial selection during domestication.

\section{Discussion}

Our results establish a connection between ancient polyploidy and contemporary diversity and domestication in crop varieties of Brassica rapa. Although polyploidy has long been hypothesized to be important for crop domestication, 
phylogenetic analyses have only recently confirmed that it is a key factor (Salman-Minkov et al., 2016). However, the mechanisms linking polyploidy and domestication itself have remained unresolved. Our results provide support for at least one genetic mechanism linking polyploidy and domestication. Paleologs, genes retained from an ancient polyploidy, were enriched in our candidate gene lists for crops of $B$. rapa. They were enriched in all three of our lists from genome scans using different statistical approaches, as well as a list developed from fine mapping and other genetic studies in the literature. In the case of $B$. rapa, the polyploid event occurred 9-28 MYA (Lukens et al., 2004; Lysak et al., 2005; Beilstein et al., 2010; Wang et al., 2011; Arias et al., 2014; Cheng et al., 2014; Franzke et al., 2016), long before the crop varieties were domesticated by humans. Many of these crop varieties were only domesticated in the last few thousand years (Qi et al., 2017), but we still find that paleologs are over-represented in a diverse range of candidate gene lists. This suggests that even ancient polyploidy may contribute to domestication and potentially adaptation long after genomes have diploidized.

We also found that paleolog retention patterns were important during domestication. Paleologs that were retained as singletons or in duplicate were enriched in candidate gene lists that involved significant changes in protein evolution and differential gene expression. In contrast, paleologs retained in triplicate from the Brassiceae mesohexaploidy were only enriched in candidate gene lists with significant differential expression changes, but not in those with significant protein changes during domestication. These results are consistent with the dosage balance hypothesis. Genes that retain all copies to maintain dosage balance are expected to be more constrained to continue making the same protein, whereas genes that are not retained to maintain dosage-balance are expected to have less constraint on their protein coding sequences (Freeling, 2009; Jiang et al., 2013; Conant et al., 2014; Blischak et al., 2016; Defoort et al., 2019). Although analyses find that some genes returning to single-copy following WGD are also highly constrained (De Smet et al., 2013; Li et al., 2016), our candidate gene lists were enriched with single copy 
paleologs across all of our analyses. This is likely because our single-copy list includes this set of "core" genes under strong constraints that returned to single-copy rapidly following WGD as well as those that returned to single-copy more gradually over millions of years. Indeed, our single-copy paleologs are enriched with the same types of GO terms as previously identified “core” single-copy genes (Hao et al., 2020), so our list does include the types of genes we expect to quickly return to single-copy (De Smet et al., 2013; Li et al., 2016). The gradient of paleolog retention categories following the Brassiceae mesohexaploidy-single, double, or triple retained genes-may produce more variation in the resolution of genes that would only be able to return to single copy following a WGD. The paleologs retained in triplicate, in particular, provide a strong case to test the biology of genes under apparently strong dosage balance. Overall, the patterns of paleolog retention and their roles in the domestication of $B$. rapa appear to follow the rules of the dosage-balance hypothesis.

Why are paleologs over-represented among the candidate genes for $B$. rapa domestication and crop improvement? Our results suggest that it may be because this class of genes harbors more genetic diversity than other genes in the genome. Across all of our analyses, paleologs consistently had significantly more nucleotide diversity than non-paleologs in B. rapa. Considering that many paleologs have been maintained in duplicate or triplicate for many generations since the mesohexaploidy that occurred 9-28 MYA, relaxed selection may yield elevated diversity at these genes as observed in other studies of paralog evolution (Lynch \& Conery, 2000; Kondrashov et al., 2002; Conant \& Wagner, 2003; Brunet et al., 2006; Aagaard et al., 2006; Scannell \& Wolfe, 2008; Shan et al., 2009; Viaene et al., 2009; Innan \& Kondrashov, 2010; Lee \& Irish, 2011; Ascencio et al., 2017). Consistent with relaxed selection, we found that nucleotide diversity was elevated for both synonymous and nonsynonymous substitutions in $B$. rapa paleologs compared to non-paleologs. Notably, nucleotide diversity was higher across all categories of paleologs in our analyses, including paleologs that are now retained in single copy. This suggests that the higher diversity could be a legacy of past duplication rather than a product of ongoing masking at some 
paleolog loci. It may also be that non-paleologs are under more constraint than paleologs. However, we still observed a significant difference in diversity even in our POInT analysis where nearly $2 / 3$ of the genome was classified as non-paleologs, suggesting that the difference is probably not entirely due to extraordinary constraint and purifying selection on non-paleologs. Further research is needed to better understand why the paleologs in $B$. rapa have elevated nucleotide diversity compared to the non-paleologs. The elevated diversity in the paleologs does not appear to be an artifact of read mapping error given that we recovered the same pattern with two different read mapping approaches and observed no difference when using only uniquely mapped reads. Relatively long Illumina read lengths (150 bp) and the nearly $30 \%$ synonymous divergence of paleologs from the Brassiceae mesohexaploidy may have limited read mapping errors caused by paralogy. Notably, the most recent paralogs were classified as "non-paleologs” together with other genes derived from duplication events other than polyploidy. Read mapping error rates would be expected to be the highest among these recent paralogs, but we consistently observed across our analyses that estimates of nucleotide diversity were significantly lower among non-paleologs than the paleologs. Regardless of the cause of the increased variation, it does not appear to be an artifact of read mapping and may explain why these genes are over-represented in the candidate gene lists. Increased genetic diversity is expected to be associated with greater phenotypic variation that could be selected during domestication. Paleologs may be over-represented in our candidate gene lists simply because they contain more genetic diversity than non-paleologs in B. rapa. Although many paleopolyploid plant genomes have been analyzed, this difference in genetic diversity has not yet been observed to the best of our knowledge.

The presence of elevated genetic variation in $B$. rapa paleologs suggests that domestication and crop improvement may have proceeded largely from standing variation. Rapid responses to selection, such as the response to selection to domestication and crop improvement, will proceed much faster if standing genetic diversity is high enough to facilitate simultaneous selection at multiple sites (Barrett \& 
Schluter, 2008; Messer \& Petrov, 2013; Matuszewski et al., 2015). It is an open question how much domestication has proceeded from standing genetic diversity, but a recent analysis suggests that maize could have been domesticated from standing variation in teosinte (Yang et al., 2019). There are also examples of candidate genes associated with crops, including in Brassica oleracea (Purugganan et al., 2000), that appear to be selected from standing genetic diversity. Given the elevated diversity of paleologs in B. rapa, we may expect domestication in these crops to be dominated by soft sweeps (Messer \& Petrov, 2013; Hermisson \& Pennings, 2017). A complicating factor in characterizing hard and soft sweeps in B. rapa at the moment is the significant difference in genetic variation between paleologs and non-paleologs that may skew such diversity based analyses. More sophisticated approaches that leverage recent advances in deep learning (Schrider \& Kern, 2018; Kern \& Schrider, 2018; Flagel et al., 2019) that are trained to account for differences in variation because of gene origins will likely overcome these issues. Ultimately, this will allow us to characterize the genetics of domestication in the diverse crop varieties of $B$. rapa while providing new insight into how paleopolyploidy may influence the architecture of adaptation in plant genomes.

The ancient hexaploidy in the Brassiceae has been hypothesized to be the source of the outstanding diversity of Brassica crop varieties (Lukens et al., 2004; Cheng et al., 2014, 2016). Our results support this hypothesis by finding that candidate genes for domestication of $B$. rapa crop varieties are enriched in regions of the genome duplicated in the mesohexaploidy. It remains to be seen if paleologs are also significantly enriched in the candidate domestication genes in the crops of other Brassica species. Other types of genetic variation, such as structural variants like presence/absence and copy number variants, may also be influenced by post-polyploid genome evolution and play a role in domestication (Golicz et al., 2016; Song et al., 2020; Gabur et al., 2020). Developing pan-genome resources (Bayer et al., 2020) for $B$. rapa would allow us to better assess diversity across the crop varieties, and test how different types of genetic diversity have contributed to domestication. Brassica are 
known as the dogs of the plant world for the incredible morphological diversity and number of different domesticated crops compared to other plants (Cheng et al., 2014; Liu et al., 2014; An et al., 2019). Experimental evolution studies in B. rapa have also been able to rapidly select for new pollination syndromes (Gervasi \& Schiestl, 2017; Schiestl et al., 2018). The higher genetic diversity of paleologs in Brassica may play a significant role in this morphological diversity and the rapid responses to selection. However, paleopolyploidy is common among flowering plants with the average species experiencing nearly five rounds of WGD in its ancestry (One Thousand Plant Transcriptomes Initiative, 2019; Li \& Barker, 2020). If elevated variation in paleologs is a general phenomenon, then we would expect it to be important in the domestication of many other crops and it may not completely explain the outstanding diversity of Brassica crops. Further analyses of paleolog variation and enrichment in other crops are needed to understand the generality of our findings and the implications for the domestication of diverse crops like in Brassica. Presently, our results provide a testable hypothesis to explain the observed correlation of polyploidy and crop domestication (Salman-Minkov et al., 2016).

More broadly, our results suggest that paleopolyploidy may leave behind a legacy of elevated genetic diversity across the duplicated remnants of diploidized genomes. Although most models and studies of polyploid evolution compare diploids and polyploids (Otto \& Whitton, 2000; Ramsey \& Schemske, 2002; Otto, 2007; Selmecki et al., 2015; Laport \& Ng, 2017; Paape et al., 2018; Monnahan et al., 2019; Han et al., 2019; Baniaga et al., 2020), our comparison of paleologs and non-paleologs within a diploidized paleopolyploid uncovered evidence for similar dynamics ongoing within plant genomes even millions of years after whole genome duplication. The extensive genome duplication history of plants may result in genomes with different levels of diversity based on the mechanisms of gene origin and retention. Although this remains to be broadly tested, it raises a few testable predictions. If this is a general phenomenon in plants, then we may expect that diploid plants with more paleologs will have higher genetic diversity than those with fewer paleologs. As paleologs are lost 
over time due to fractionation and gene turnover, we would also expect genetic diversity in diploid plants to be correlated with the time since their most recent paleopolyploid event. The long-term effects of paleopolyploidy on genetic diversity observed here in $B$. rapa may also help explain a broader phenomenon, the lag time of paleopolyploidy and diversification (Schranz et al., 2012). Recent research has found that paleopolyploidy in plants is associated with diversification rate increases, but these rate increases often occur many millions of years following WGDs (Landis et al., 2018). If relatively high genetic diversity is important for the adaptation and expansion that leads to macroevolutionary signatures of net diversification rate increases, then the observed lag between diversification and polyploidy observed in many studies could be explained by the lasting increase in diversity at paleologs. Although our analyses are centered on how ancient polyploidy contributed to the diversity of $B$. rapa, additional analyses in other plants will provide crucial data to test the new hypotheses described above. Given the distribution of polyploidy throughout the history of flowering plants (One Thousand Plant Transcriptomes Initiative, 2019), our results suggest that the genetic legacy of these WGDs could contribute to the diversity and adaptation of plants millions of years later.

\section{Acknowledgements}

We thank A. Baniaga, G. Finch, Z. Li, and B. Sutherland for feedback and comments on drafts of this manuscript. Hosting infrastructure and services provided by the Biotechnology Computing Facility (BCF) at the University of Arizona. This research was supported by NSF-IOS-1339156 to M.S.B., G.C.C., and J.C.P.

\section{Author Contributions}

H.A. conducted the RNA experiments. X.Q. conducted gene age distribution, candidate gene survey, and all statistics. X.Q. and T.E.H. conducted the differential gene expression analyses. X.Q., C.D., M.T.W.M., and P.D.B. conducted the gene diversity 
analyses. X.Q. and M.S.B. designed the experiments and wrote the manuscript with input from all co-authors. All authors read and approved the manuscript.

\section{Competing Financial Interests}

The authors declare no competing financial interests.

Data availability. All the data sets generated during the current study are available in the NCBI Sequence Read Archive (SRA) under accession number SRP072186 (http://www.ncbi.nlm.nih.gov/sra/SRP072186). The accession numbers are summarized in Supplementary Table 2.

\section{References}

Aagaard JE, Willis JH, Phillips PC. 2006. Relaxed selection among duplicate floral regulatory genes in Lamiales. Journal of Molecular Evolution 63: 493-503.

Anderson E. 1969. Plants, Man and Life. University of California Press.

An H, Qi X, Gaynor ML, Hao Y, Gebken SC, Mabry ME, McAlvay AC, Teakle GR, Conant GC, Barker MS, et al. 2019. Transcriptome and organellar sequencing highlights the complex origin and diversification of allotetraploid Brassica napus. Nature Communications 10: 2878.

Arias T, Beilstein MA, Tang M, McKain MR, Pires JC. 2014. Diversification times among Brassica (Brassicaceae) crops suggest hybrid formation after 20 million years of divergence. American Journal of Botany 101: 86-91.

Arrigo N, Barker MS. 2012. Rarely successful polyploids and their legacy in plant genomes. Current Opinion in Plant Biology 15: 140-146.

Ascencio D, Ochoa S, Delaye L, DeLuna A. 2017. Increased rates of protein evolution and asymmetric deceleration after the whole-genome duplication in yeasts. BMC Evolutionary Biology 17: 40.

Baduel P, Bray S, Vallejo-Marin M. 2018. The‘ Polyploid Hop': shifting challenges and opportunities over the evolutionary lifespan of genome duplications. Frontiers in Ecology and the Environment.

Baniaga AE, Marx HE, Arrigo N, Barker MS. 2020. Polyploid plants have faster rates of multivariate niche differentiation than their diploid relatives. Ecology Letters 23: 68-78.

Barker MS, Dlugosch KM, Dinh L, Challa RS, Kane NC, King MG, Rieseberg LH. 2010. 
EvoPipes. net: bioinformatic tools for ecological and evolutionary genomics. Evolutionary Bioinformatics Online 6: 143.

Barker MS, Kane NC, Matvienko M, Kozik A, Michelmore RW, Knapp SJ, Rieseberg LH. 2008. Multiple paleopolyploidizations during the evolution of the Compositae reveal parallel patterns of duplicate gene retention after millions of years. Molecular Biology and Evolution 25: 2445-2455.

Barker MS, Vogel H, Schranz ME. 2009. Paleopolyploidy in the Brassicales: analyses of the Cleome transcriptome elucidate the history of genome duplications in Arabidopsis and other Brassicales. Genome Biology and Evolution 1: 391-399.

Barrett RDH, Schluter D. 2008. Adaptation from standing genetic variation. Trends in Ecology \& Evolution 23: 38-44.

Bayer PE, Golicz AA, Scheben A, Batley J, Edwards D. 2020. Plant pan-genomes are the new reference. Nature Plants 6:914-920.

Beilstein MA, Nagalingum NS, Clements MD, Manchester SR, Mathews S. 2010. Dated molecular phylogenies indicate a Miocene origin for Arabidopsis thaliana. Proceedings of the National Academy of Sciences of the United States of America 107: 18724-18728.

Blischak PD, Mabry ME, Conant GC, Chris Pires J. 2016. Integrating Networks, Phylogenomics, and Population Genomics for the Study of Polyploidy. Annual Review of Ecology, Evolution, and Systematics 49: 253-278.

Bolger AM, Lohse M, Usadel B. 2014. Trimmomatic: a flexible trimmer for Illumina sequence data. Bioinformatics 30: 2114-2120.

Brunet FG, Roest Crollius H, Paris M, Aury J-M, Gibert P, Jaillon O, Laudet V, Robinson-Rechavi M. 2006. Gene loss and evolutionary rates following whole-genome duplication in teleost fishes. Molecular Biology and Evolution 23: 1808-1816.

Cheng F, Mandáková T, Wu J, Xie Q, Lysak MA, Wang X. 2013. Deciphering the diploid ancestral genome of the Mesohexaploid Brassica rapa. The Plant Cell 25: 1541-1554.

Cheng F, Sun R, Hou X, Zheng H, Zhang F, Zhang Y, Liu B, Liang J, Zhuang M, Liu Y, et al. 2016. Subgenome parallel selection is associated with morphotype diversification and convergent crop domestication in Brassica rapa and Brassica oleracea. Nature Genetics 48:1218-1224.

Cheng F, Wu J, Fang L, Sun S, Liu B, Lin K, Bonnema G, Wang X. 2012. Biased gene fractionation and dominant gene expression among the subgenomes of Brassica rapa. PloS one 7: e36442.

Cheng F, Wu J, Wang X. 2014. Genome triplication drove the diversification of Brassica plants. Horticulture research 1: 14024.

Cingolani P, Platts A, Wang LL, Coon M, Nguyen T, Wang L, Land SJ, Lu X, Ruden DM. 2012. A program for annotating and predicting the effects of single nucleotide polymorphisms, 
SnpEff: SNPs in the genome of Drosophila melanogaster strain w1118; iso-2; iso-3. Fly 6: 80-92.

Conant GC, Birchler JA, Pires JC. 2014. Dosage, duplication, and diploidization: clarifying the interplay of multiple models for duplicate gene evolution over time. Current Opinion in Plant Biology 19: 91-98.

Conant GC, Wagner A. 2003. Asymmetric sequence divergence of duplicate genes. Genome Research 13: 2052-2058.

Danecek P, Auton A, Abecasis G, Albers CA, Banks E, DePristo MA, Handsaker RE, Lunter G, Marth GT, Sherry ST, et al. 2011. The variant call format and VCFtools. Bioinformatics 27: 2156-2158.

Defoort J, Van de Peer Y, Carretero-Paulet L. 2019. The Evolution of Gene Duplicates in Angiosperms and the Impact of Protein-Protein Interactions and the Mechanism of Duplication. Genome Biology and Evolution 11: 2292-2305.

De Smet R, Adams KL, Vandepoele K, Van Montagu MCE, Maere S, Van de Peer Y. 2013. Convergent gene loss following gene and genome duplications creates single-copy families in flowering plants. Proceedings of the National Academy of Sciences of the United States of America 110: 2898-2903.

Dobin A, Davis CA, Schlesinger F, Drenkow J, Zaleski C, Jha S, Batut P, Chaisson M, Gingeras TR. 2013. STAR: ultrafast universal RNA-seq aligner. Bioinformatics 29: 15-21.

Flagel L, Brandvain Y, Schrider DR. 2019. The Unreasonable Effectiveness of Convolutional Neural Networks in Population Genetic Inference. Molecular Biology and Evolution 36: 220-238.

Franzke A, Koch MA, Mummenhoff K. 2016. Turnip Time Travels: Age Estimates in Brassicaceae. Trends in Plant Science 21: 554-561.

Freeling M. 2009. Bias in plant gene content following different sorts of duplication: tandem, whole-genome, segmental, or by transposition. Annual Review of Plant Biology 60: 433-453.

Fu W, Ye X, Ren J, Li Q, Du J, Hou A, Mei F, Feng H, Liu Z. 2019. Fine mapping of lcm1, a gene conferring chlorophyll-deficient golden leaf in Chinese cabbage (Brassica rapa ssp.

pekinensis). Molecular Breeding: new strategies in plant improvement 39: 52.

Gabur I, Chawla HS, Lopisso DT, von Tiedemann A, Snowdon RJ, Obermeier C. 2020. Gene presence-absence variation associates with quantitative Verticillium longisporum disease resistance in Brassica napus. Scientific Reports 10: 4131.

Gervasi DDL, Schiestl FP. 2017. Real-time divergent evolution in plants driven by pollinators. Nature Communications 8: 14691.

Goff L, Trapnell C, Kelley D. 2012. cummeRbund: Analysis, exploration, manipulation, and visualization of Cufflinks high-throughput sequencing data. $R$ package version.

Golicz AA, Bayer PE, Barker GC, Edger PP, Kim H, Martinez PA, Chan CKK, Severn-Ellis A, 
McCombie WR, Parkin IAP, et al. 2016. The pangenome of an agronomically important crop plant Brassica oleracea. Nature Communications 7: 13390.

Han T-S, Zheng Q-J, Onstein RE, Rojas-Andrés BM, Hauenschild F, Muellner-Riehl AN, Xing Y-W. 2019. Polyploidy promotes species diversification of Allium through ecological shifts. The New Phytologist.

Hao Y, Mabry ME, Edger P, Freeling M, Zheng C, Jin L, VanBuren R, Colle M, An H, Shawn Abrahams R, et al. 2020. The Contributions of the Allopolyploid Parents of the Mesopolyploid Brassiceae are Evolutionarily Distinct but Functionally Compatible. bioRxiv 2020.08.10.245258.

Heiser CB. 1990. Seed to Civilization: The Story of Food. Harvard University Press.

Hermisson J, Pennings PS. 2017. Soft sweeps and beyond: understanding the patterns and probabilities of selection footprints under rapid adaptation ( $\mathrm{J}$ Kelley, Ed.). Methods in Ecology and Evolution 8: 700-716.

Hilu KW. 1993. Polyploidy and the Evolution of Domesticated Plants. American Journal of Botany 80: 1494-1499.

Huang Z, Peng G, Gossen BD, Yu F. 2019. Fine mapping of a clubroot resistance gene from turnip using SNP markers identified from bulked segregant RNA-Seq. Molecular Breeding: new strategies in plant improvement 39: 131.

Innan H, Kondrashov F. 2010. The evolution of gene duplications: classifying and distinguishing between models. Nature Reviews Genetics 11: 97-108.

Jiang W-K, Liu Y-L, Xia E-H, Gao L-Z. 2013. Prevalent role of gene features in determining evolutionary fates of whole-genome duplication duplicated genes in flowering plants. Plant Physiology 161: 1844-1861.

Kato T, Morita MT, Fukaki H, Yamauchi Y, Uehara M, Niihama M, Tasaka M. 2002. SGR2, a phospholipase-like protein, and ZIG/SGR4, a SNARE, are involved in the shoot gravitropism of Arabidopsis. The Plant Cell 14: 33-46.

Kern AD, Schrider DR. 2018. diploS/HIC: An Updated Approach to Classifying Selective Sweeps. G3 8: 1959-1970.

Kondrashov FA, Rogozin IB, Wolf YI, Koonin EV. 2002. Selection in the evolution of gene duplications. Genome biology 3: RESEARCH0008.

Korneliussen TS, Albrechtsen A, Nielsen R. 2014. ANGSD: Analysis of Next Generation Sequencing Data. BMC Bioinformatics 15: 356.

Korneliussen TS, Moltke I, Albrechtsen A, Nielsen R. 2013. Calculation of Tajima's D and other neutrality test statistics from low depth next-generation sequencing data. $B M C$

Bioinformatics 14: 289.

Landis JB, Soltis DE, Li Z, Marx HE, Barker MS, Tank DC, Soltis PS. 2018. Impact of whole-genome duplication events on diversification rates in angiosperms. American Journal of 
Botany 105: 348-363.

Laport RG, Ng J. 2017. Out of one, many: The biodiversity considerations of polyploidy. American Journal of Botany 104:1119-1121.

Lee H-L, Irish VF. 2011. Gene duplication and loss in a MADS box gene transcription factor circuit. Molecular Biology and Evolution 28: 3367-3380.

Levin DA. 1983. Polyploidy and Novelty in Flowering Plants. The American Naturalist 122: $1-25$.

Lewis WH. 1980. Polyploidy in Species Populations. In: Lewis WH, ed. Basic Life Sciences. Polyploidy: Biological Relevance. Boston, MA: Springer US, 103-144.

Li H. 2011. A statistical framework for SNP calling, mutation discovery, association mapping and population genetical parameter estimation from sequencing data. Bioinformatics 27: 2987-2993.

Li Z, Barker MS. 2020. Inferring putative ancient whole-genome duplications in the 1000 Plants (1KP) initiative: access to gene family phylogenies and age distributions. GigaScience 9.

Li Z, Defoort J, Tasdighian S, Maere S, Van de Peer Y, De Smet R. 2016. Gene Duplicability of Core Genes Is Highly Consistent across All Angiosperms. The Plant Cell 28: 326-344.

Li H, Handsaker B, Wysoker A, Fennell T, Ruan J, Homer N, Marth G, Abecasis G, Durbin R, 1000 Genome Project Data Processing Subgroup. 2009. The Sequence Alignment/Map format and SAMtools. Bioinformatics 25: 2078-2079.

Li J, Liu B, Cheng F, Wang X, Aarts MGM, Wu J. 2014. Expression profiling reveals functionally redundant multiple-copy genes related to zinc, iron and cadmium responses in Brassica rapa. The New Phytologist 203: 182-194.

Liu S, Liu Y, Yang X, Tong C, Edwards D, Parkin IAP, Zhao M, Ma J, Yu J, Huang S, et al. 2014. The Brassica oleracea genome reveals the asymmetrical evolution of polyploid genomes. Nature Communications 5: 3930.

Lukens LN, Quijada PA, Udall J, Pires JC, Schranz ME, Osborn TC. 2004. Genome redundancy and plasticity within ancient and recent Brassica crop species: BRASSICA GENOME STRUCTURE AND PLASTICITY. Biological journal of the Linnean Society. Linnean Society of London 82: 665-674.

Lynch M, Conery JS. 2000. The evolutionary fate and consequences of duplicate genes. Science 290: 1151-1155.

Lysak MA, Koch MA, Pecinka A, Schubert I. 2005. Chromosome triplication found across the tribe Brassiceae. Genome Research 15: 516-525.

Mano E, Horiguchi G, Tsukaya H. 2006. Gravitropism in leaves of Arabidopsis thaliana (L.) Heynh. Plant \& Cell Physiology 47: 217-223.

Matuszewski S, Hermisson J, Kopp M. 2015. Catch Me if You Can: Adaptation from Standing 
Genetic Variation to a Moving Phenotypic Optimum. Genetics 200: 1255-1274.

McDonald JH, Kreitman M. 1991. Adaptive protein evolution at the Adh locus in Drosophila. Nature 351: 652-654.

McLachlan GJ, Peel D, Basford KE, Adams P. 1999. The EMMIX software for the fitting of mixtures of normal and t-components. Journal of statistical software 4: 1-14.

Messer PW, Petrov DA. 2013. Population genomics of rapid adaptation by soft selective sweeps. Trends in Ecology \& Evolution 28: 659-669.

Meyer RS, DuVal AE, Jensen HR. 2012. Patterns and processes in crop domestication: an historical review and quantitative analysis of 203 global food crops. The New Phytologist 196: 29-48.

Monnahan P, Kolár̆ F, Baduel P, Sailer C, Koch J, Horvath R, Laenen B, Schmickl R, Paajanen P, Šrámková G, et al. 2019. Pervasive population genomic consequences of genome duplication in Arabidopsis arenosa. Nature Ecology \& Evolution 3: 457-468.

Nielsen R, Korneliussen T, Albrechtsen A, Li Y, Wang J. 2012. SNP calling, genotype calling, and sample allele frequency estimation from New-Generation Sequencing data. PloS One 7: e37558.

One Thousand Plant Transcriptomes Initiative. 2019. One thousand plant transcriptomes and the phylogenomics of green plants. Nature 574: 679-685.

Otto SP. 2007. The evolutionary consequences of polyploidy. Cell 131: 452-462.

Otto SP, Whitton J. 2000. Polyploid incidence and evolution. Annual Review of Genetics 34: 401-437.

Paape T, Briskine RV, Halstead-Nussloch G, Lischer HEL, Shimizu-Inatsugi R, Hatakeyama M, Tanaka K, Nishiyama T, Sabirov R, Sese J, et al. 2018. Patterns of polymorphism and selection in the subgenomes of the allopolyploid Arabidopsis kamchatica. Nature Communications 9: 3909.

Paterson AH. 2005. Polyploidy, evolutionary opportunity, and crop adaptation. Genetica 123: 191-196.

Pavlidis P, Živkovic D, Stamatakis A, Alachiotis N. 2013. SweeD: likelihood-based detection of selective sweeps in thousands of genomes. Molecular Biology and Evolution 30: 2224-2234.

Purugganan MD, Boyles AL, Suddith JI. 2000. Variation and selection at the CAULIFLOWER floral homeotic gene accompanying the evolution of domesticated Brassica oleracea. Genetics 155: 855-862.

Qi X, An H, Ragsdale A, Hall TE, Gutenkunst RN, Pires JC, Barker MS. 2017. Genomic inferences of domestication events are corroborated by written records in Brassica rapa. Molecular Ecology 26: 3373-3388.

Ramsey J, Schemske DW. 2002. Neopolyploidy in Flowering Plants. Annual Review of Ecology 
and Systematics 33: 589-639.

R Core Team. 2019. R: A language and environment for statistical computing.

Renny-Byfield S, Wendel JF. 2014. Doubling down on genomes: polyploidy and crop plants. American Journal of Botany 101: 1711-1725.

Salman-Minkov A, Sabath N, Mayrose I. 2016. Whole-genome duplication as a key factor in crop domestication. Nature Plants 2: 16115.

Scannell DR, Wolfe KH. 2008. A burst of protein sequence evolution and a prolonged period of asymmetric evolution follow gene duplication in yeast. Genome Research 18: 137-147.

Schiestl FP, Balmer A, Gervasi DD. 2018. Real-time evolution supports a unique trajectory for generalized pollination. Evolution 72: 2653-2668.

Schranz ME, Mohammadin S, Edger PP. 2012. Ancient whole genome duplications, novelty and diversification: the WGD Radiation Lag-Time Model. Current Opinion in Plant Biology 15: 147-153.

Schrider DR, Kern AD. 2018. Supervised Machine Learning for Population Genetics: A New Paradigm. Trends in Genetics 34: 301-312.

Selmecki AM, Maruvka YE, Richmond PA, Guillet M, Shoresh N, Sorenson AL, De S, Kishony R, Michor F, Dowell R, et al. 2015. Polyploidy can drive rapid adaptation in yeast. Nature 519: 349-352.

Shan H, Zahn L, Guindon S, Wall PK, Kong H, Ma H, DePamphilis CW, Leebens-Mack J. 2009. Evolution of plant MADS box transcription factors: evidence for shifts in selection associated with early angiosperm diversification and concerted gene duplications. Molecular Biology and Evolution 26: 2229-2244.

Shimizu-Inatsugi R, Terada A, Hirose K, Kudoh H, Sese J, Shimizu KK. 2017. Plant adaptive radiation mediated by polyploid plasticity in transcriptomes. Molecular Ecology 26: 193-207.

Song J-M, Guan Z, Hu J, Guo C, Yang Z, Wang S, Liu D, Wang B, Lu S, Zhou R, et al. 2020. Eight high-quality genomes reveal pan-genome architecture and ecotype differentiation of Brassica napus. Nature Plants 6: 34-45.

Szklarczyk D, Gable AL, Lyon D, Junge A, Wyder S, Huerta-Cepas J, Simonovic M, Doncheva NT, Morris JH, Bork P, et al. 2019. STRING v11: protein-protein association networks with increased coverage, supporting functional discovery in genome-wide experimental datasets. Nucleic Acids Research 47: D607-D613.

Tang H, Lyons E, Pedersen B, Schnable JC, Paterson AH, Freeling M. 2011. Screening synteny blocks in pairwise genome comparisons through integer programming. $B M C$ Bioinformatics 12: 102.

Tiley GP, Barker MS, Burleigh JG. 2018. Assessing the performance of Ks plots for detecting ancient whole genome duplications. Genome Biology and Evolution 10: 2882-2898. 
Trapnell C, Hendrickson DG, Sauvageau M, Goff L, Rinn JL, Pachter L. 2013. Differential analysis of gene regulation at transcript resolution with RNA-seq. Nature Biotechnology 31: 46-53.

Trapnell C, Pachter L, Salzberg SL. 2009. TopHat: discovering splice junctions with RNA-Seq. Bioinformatics 25: 1105-1111.

Trapnell C, Roberts A, Goff L, Pertea G, Kim D, Kelley DR, Pimentel H, Salzberg SL, Rinn JL, Pachter L. 2012. Differential gene and transcript expression analysis of RNA-seq experiments with TopHat and Cufflinks. Nature Protocols 7: 562-578.

Trapnell C, Williams BA, Pertea G, Mortazavi A, Kwan G, van Baren MJ, Salzberg SL, Wold BJ, Pachter L. 2010. Transcript assembly and quantification by RNA-Seq reveals unannotated transcripts and isoform switching during cell differentiation. Nature Biotechnology 28: 511-515.

Tuteja N, Singh S, Tuteja R. 2012. Helicases in Improving Abiotic Stress Tolerance in Crop Plants. In: Tuteja N, Gill SS, Tiburcio AF, Tuteja R, eds. Improving Crop Resistance to Abiotic Stress. Weinheim, Germany: Wiley-VCH Verlag GmbH \& Co. KGaA, 435-449.

Udall JA, Wendel JF. 2006. Polyploidy and Crop Improvement. Crop Science 46: S-3-S-14.

UniProt Consortium T. 2018. UniProt: the universal protein knowledgebase. Nucleic Acids Research 46: 2699.

Viaene T, Vekemans D, Irish VF, Geeraerts A, Huysmans S, Janssens S, Smets E, Geuten K. 2009. Pistillata--duplications as a mode for floral diversification in (Basal) asterids. Molecular Biology and Evolution 26: 2627-2645.

Wang C, Li H, Li Y, Meng Q, Xie F, Xu Y, Wan Z. 2019. Genetic characterization and fine mapping BrCER4 in involved cuticular wax formation in purple cai-tai (Brassica rapa L. var. purpurea). Molecular Breeding: new strategies in plant improvement 39: 12.

Wang C, Li Y, Xie F, Kuang H, Wan Z. 2017. Cloning of the Brcer1 gene involved in cuticular wax production in a glossy mutant of non-heading Chinese cabbage (Brassica rapa L. var. communis). Molecular breeding: new strategies in plant improvement 37: 142.

Wang X, Wang H, Wang J, Sun R, Wu J, Liu S, Bai Y, Mun J-H, Bancroft I, Cheng F, et al. 2011. The genome of the mesopolyploid crop species Brassica rapa. Nature Genetics 43: 1035-1039.

Yang CJ, Samayoa LF, Bradbury PJ, Olukolu BA, Xue W, York AM, Tuholski MR, Wang W, Daskalska LL, Neumeyer MA, et al. 2019. The genetic architecture of teosinte catalyzed and constrained maize domestication. Proceedings of the National Academy of Sciences of the United States of America 116: 5643-5652.

Yu F, Zhang X, Huang Z, Chu M, Song T, Falk KC, Deora A, Chen Q, Zhang Y, McGregor L, et al. 2016. Identification of genome-wide variants and discovery of variants associated with Brassica rapa clubroot resistance gene Rcr1 through bulked segregant RNA sequencing. PloS 
bioRxiv preprint doi: https://doi org/10.1101/842351 t this version posted October 15,2020 . The copyright holder for this preprint (which was not certified by peer review) is the author/funder, who has granted bioRxiv a license to display the preprint in perpetuity. It is made available under aCC-BY 4.0 International license.

one 11: e0153218. 


\section{Figures \& Figure Legends}

Synteny + Ks B. rapa only Paleolog Inference

(a)
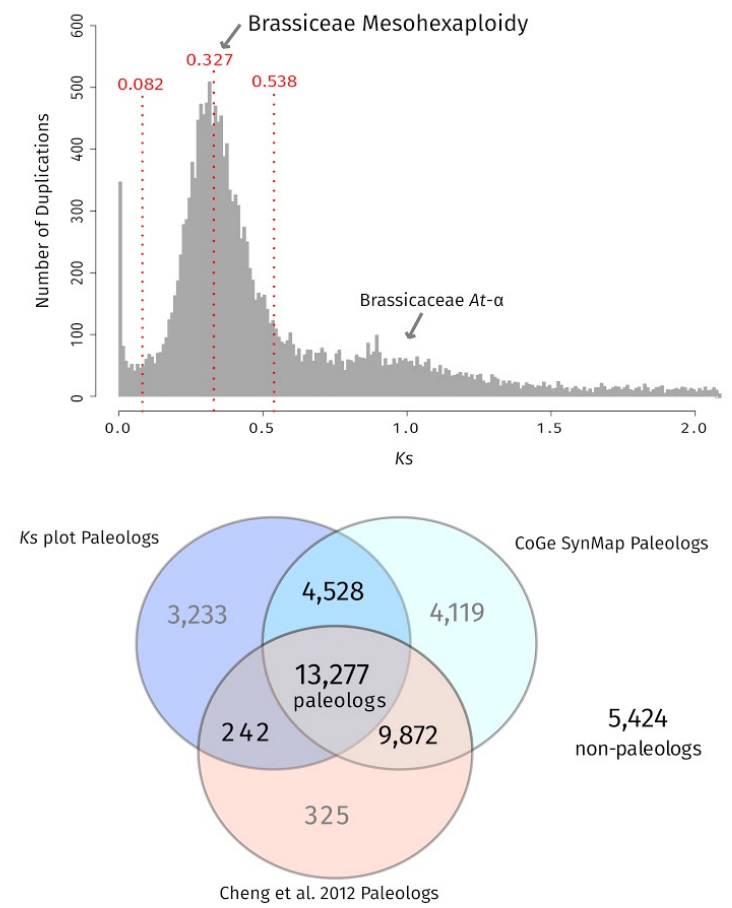

POInT Multi-Genome Paleolog Inference

(b)

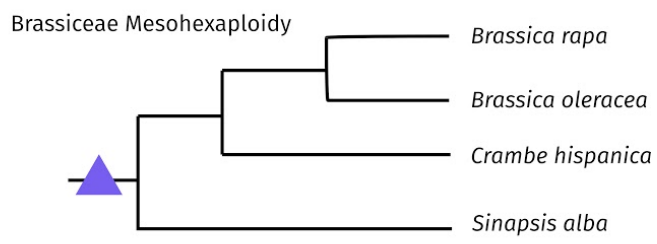

13,499 B. rapa Paleologs (>95\% confidence) 4,818 Retained as Singletons

6,128 Retained in Duplicate

2,553 Retained in Triplicate

27,527 B. rapa Non-Paleologs

Fig. 1: Identifying paleologs derived from the Brassica hexaploidy event. (a) The age distribution of gene duplications from 41,020 CDSs in the B. rapa genome (version 1.5). The $x$-axis represents the synonymous divergence of duplication events (Ks value), whereas the $y$-axis represents the number of duplications. Gene pairs with Ks divergence $0.082-0.538$ were identified as putative paleologs. Venn diagram showing the overlap among our two $B$. rapa paleolog lists with a previously reported $B$. rapa paleolog list. Genes that appeared at least twice in these three lists were considered high confidence paleologs and were used in further analyses. Genes that were not present in any of the three paleologs lists were considered to be non-paleologs. (b) Phylogeny of species's genomes used for the POInT multi-genome analysis to identify paleologs in B. rapa from Hao et al. (2020). A total of 13,499 paleologs and 27,527 non-paleologs were identified in $B$. rapa. Colored blocks indicate the retention category of paleologs and the number of genes in each category. 


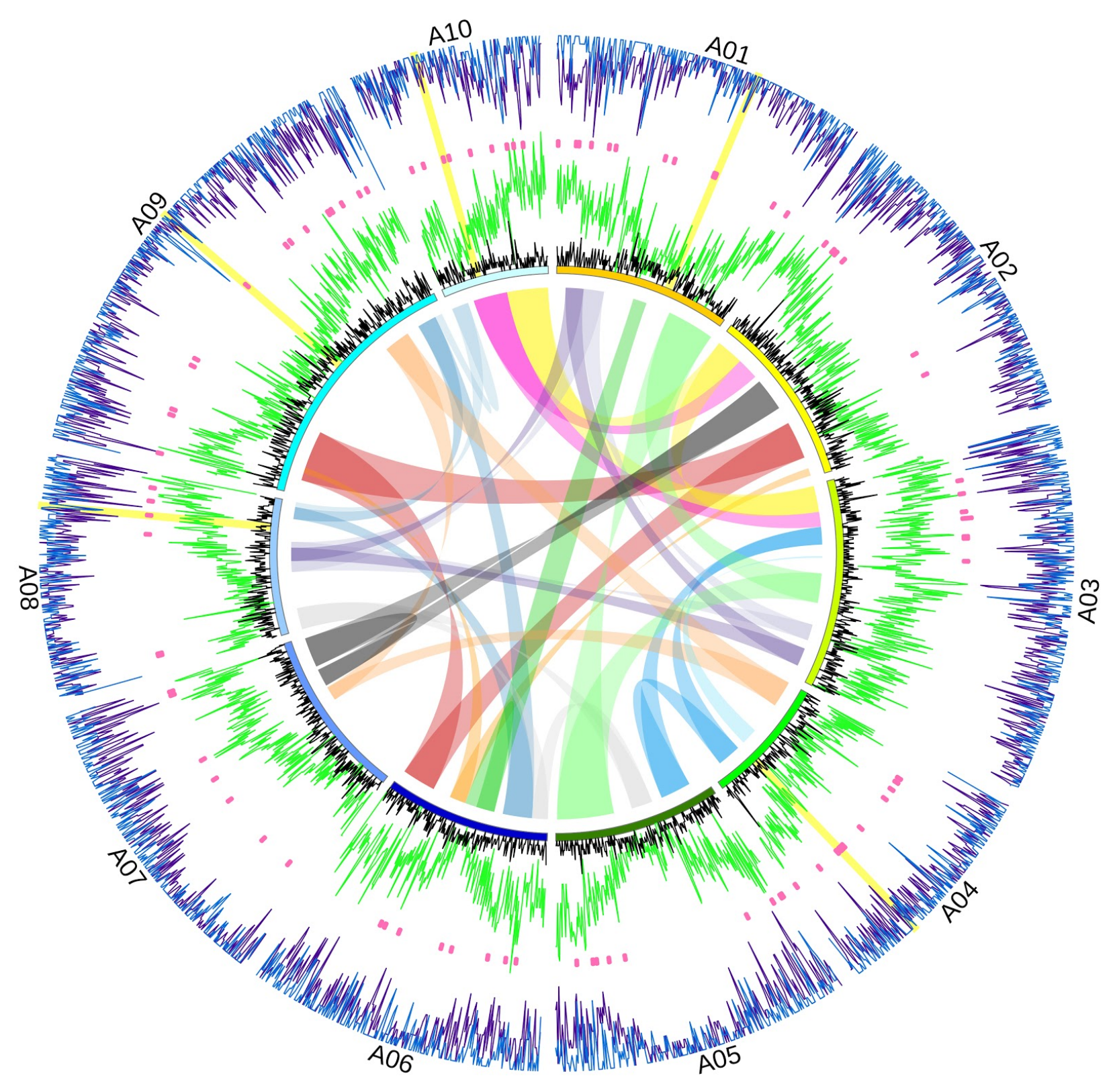

Fig. 2: Circos plot of the distribution of surveyed genes in this study. Highlighted on the outside of the plot are the Brassica hexaploidy paleologs identified by the B. rapa only synteny $+\mathrm{Ks}$ analysis (light green lines), non-paleologs (black lines), and candidate genes identified by SweeD (blue lines), the McDonald-Kreitman test (pink dots), and the significantly differentially expressed genes between the EU-CA group and the four derived B. rapa groups (purple lines). Heights of the lines indicate the 
bioRxiv preprint doi: https://doi.org/10.1101/842351; this version posted October 15,2020 . The copyright holder for this preprint (which was not certified by peer review) is the author/funder, who has granted bioRxiv a license to display the preprint in perpetuity. It is made available under aCC-BY 4.0 International license.

relative density of genes in each category in the sliding window at each location. A01-A10 represent the 10 chromosomes of $B$. rapa. The rainbow ribbons in the center represent the syntenic regions among chromosomes. The yellow bars represent the location of the five B. rapa domestication candidate genes listed in Table S5. 
(a)

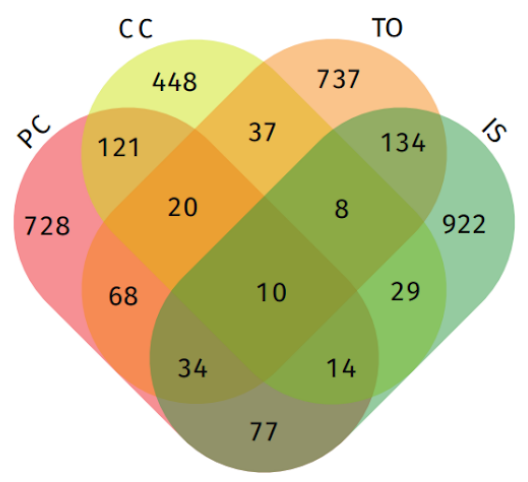

(b)

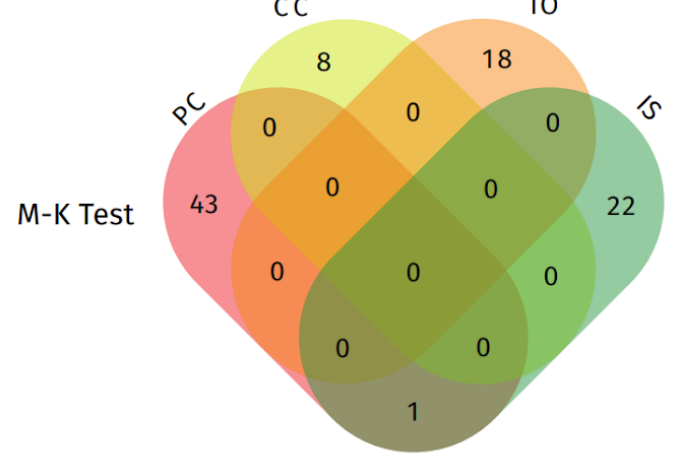

(c)

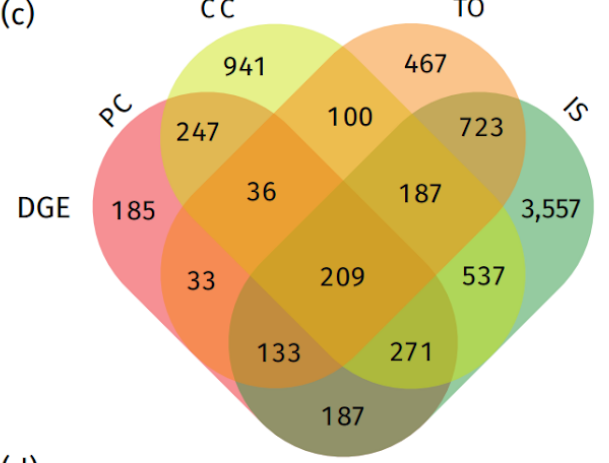

(d)

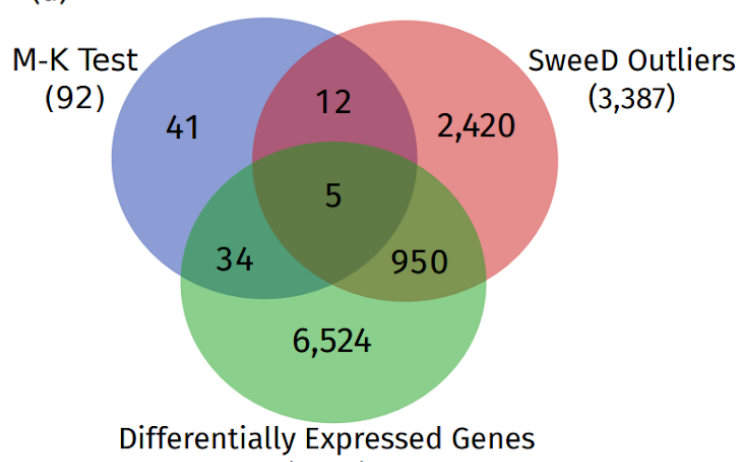

$(7,813)$

Fig. 3: Overlap among domestication candidate genes. $\mathrm{PC}=$ pak choi, $\mathrm{CC}=\mathrm{Chinese}$ cabbage, TO = toria, and IS = Indian sarson. Number and overlap of candidate genes for each crop variety inferred by (a) SweeD, (b) the McDonald-Kreitman test, and (c) differential gene expression analyses. (d) Total number and overlap of candidate genes inferred by different methods across all crop varieties. 


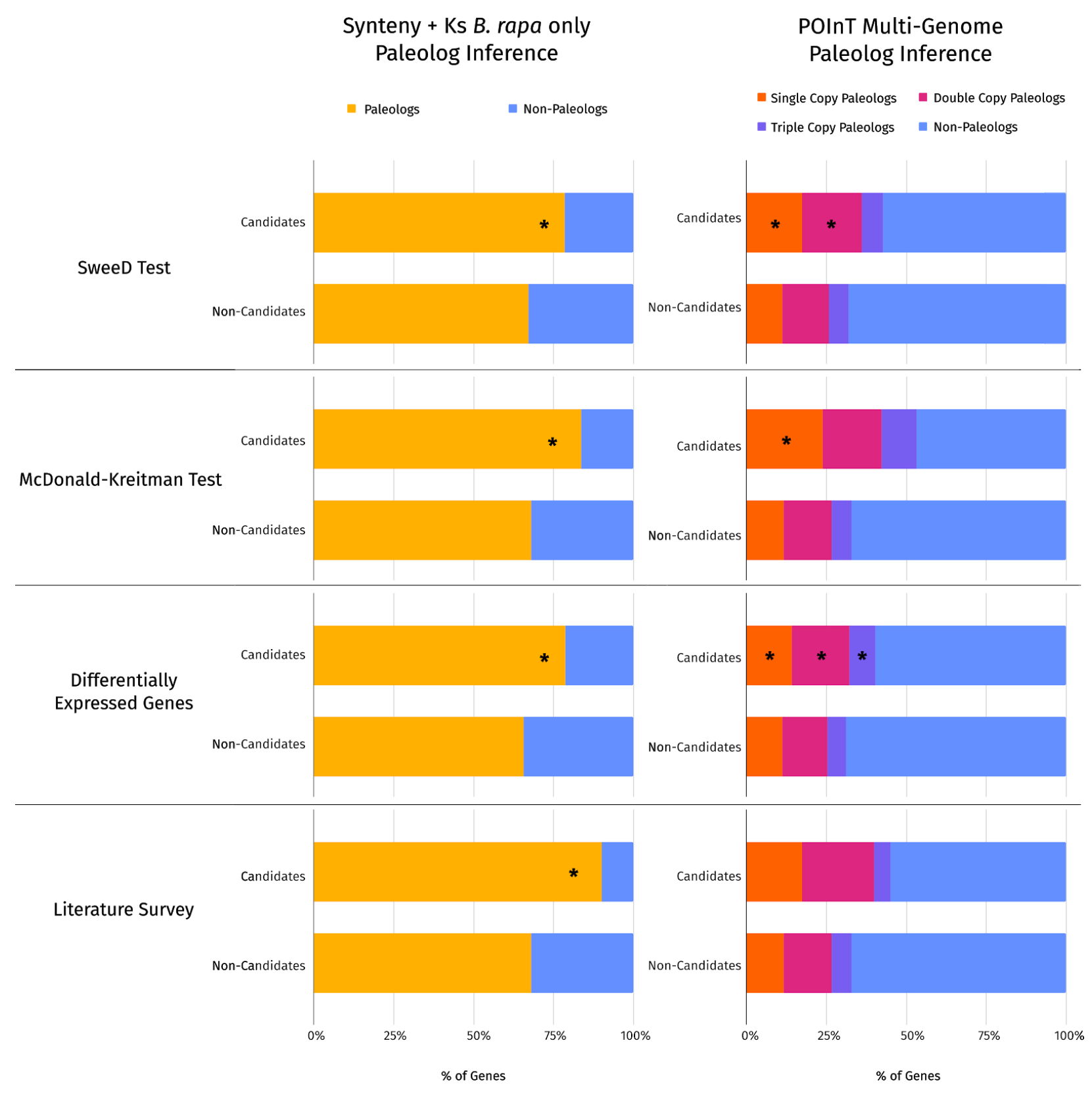

Fig. 4: Comparison of paleolog percentages across candidate gene lists. Percentage of paleologs in each candidate gene list compared to the rest of the B. rapa genome. Bar charts on the left are the paleologs (yellow) and non-paleologs (blue) identified by the $B$. rapa only synteny + Ks paleolog inference approach. Bar charts on the right are paleologs identified by the POInT multi-genome approach and include paleologs retained in single (orange), double (magenta), and triple (purple) copy as well as the 
bioRxiv preprint doi: https://doi.org/10.1101/842351; this version posted October 15,2020 . The copyright holder for this preprint (which was not certified by peer review) is the author/funder, who has granted bioRxiv a license to display the preprint in perpetuity. It is made available under aCC-BY 4.0 International license.

percentage of the B. rapa genome that are non-paleologs (blue). The asterisks indicate significant deviation from the null based on chi-square tests $(p<0.05)$. 


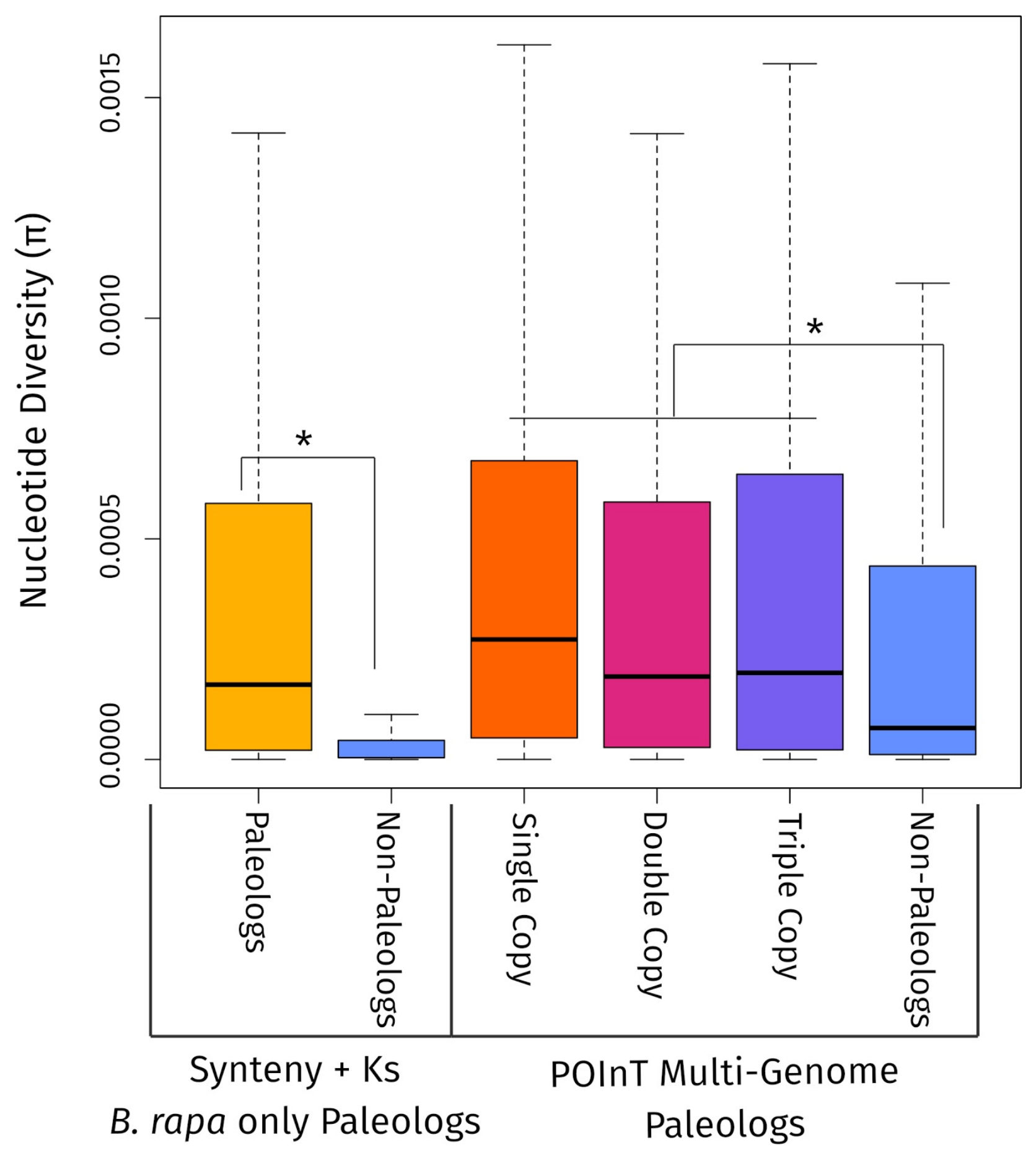

Fig. 5: Comparison of nucleotide diversity $(\pi)$ across Brassica rapa paleologs and non-paleologs. The bottom and top of each box represents the first and third quartiles, and the band inside each box is the median. Asterisks indicate significant differences between categories with a Mann-Whitney $\mathrm{U}$ test $(p<0.05)$. 


\section{Supporting Information}

Fig S1: Manhattan plots of the SweeD analyses. Each column represents a $B$. rapa genetic group: TO, toria; IS, Indian sarson; PC, pak choi; CC, Chinese cabbage. Each row represents a $B$. rapa chromosome (A01-A10). For each plot, the $x$-axis denotes the chromosome position (unit: bp), whereas the y-axis denotes the CLR value calculated in SweeD. Each dot denotes the CLR value of a genomic region. Outlier regions were indicated with red dots.

Fig S2: Circos plot of SweeD (a) and Gene Differential Expression (GDE) gene (b) density across the $B$. rapa genome. A01-A10 represent the 10 chromosomes of $B$. rapa. The four histogram layers denote the number of identified candidate genes. TO, toria (orange); IS, Indian sarson (light green); CC, Chinese cabbage (olive); PC, pak choi (red). The rainbow ribbons in the center represent the syntenic regions among chromosomes.

Fig S3: Heatmaps of expression level of genes with significantly different expression in four $B$. rapa crop varieties compared to a control group. $\mathrm{TO}=$ toria, IS $=$ Indian sarson, $\mathrm{PC}=$ pak choi, and $\mathrm{CC}=$ Chinese cabbage. The European $B$. rapa genetic group was used as control. Each column represents one $B$. rapa accession, whereas each row represents one gene with significantly different expression between the focal group and control group.

Fig. S4. Comparison of nucleotide diversity $(\pi)$ across Brassica rapa paleologs and non-paleologs from the $B$. rapa only synteny + Ks paleolog inference approach. The bottom and top of each box represents the first and third quartiles, the band inside each box is the median, and the numbers represent the mean $\pi$ of each category. Nucleotide diversity is shown for all sites, nonsynonymous sites only, and synonymous sites only for paleologs (purple shades) and non-paleologs (orange shades). 
Fig S5: ANGSD Read mapping. Nucleotide diversity in paleologs and non-paleologs based on read mapping with STAR and $\pi$ calculated from genotype likelihoods in ANGSD. Box plots on left were filtered for only uniquely mapped reads, whereas the box plots on the right included calculations of $\pi$ based on all read mappings (unfiltered). Paleologs had significantly higher levels of diversity than nonpaleologs for all read mappings (unfiltered $\mathrm{t}=48.997, \mathrm{df}=25842, \mathrm{p}<2.2 \times 10^{-16}$ for $\mathrm{t}$-test; $\mathrm{W}=$ $238,210,946$ and $p<2.2 \times 10^{-16}$ for U-test) and when filtering for only uniquely mapping reads (filtered $\mathrm{t}=44.11, \mathrm{df}=26044$, and $\mathrm{p}<2.2 \times 10^{-16}$ for $\mathrm{t}$-test; $\mathrm{W}=$ $232,582,598$ and $p<2.2 \times 10^{-16}$ for U-test), demonstrating that our results are unlikely to be affected by read mismapping.

Table S1: Brassica rapa gene IDs for genes identified as paleologs and non-paleologs in our analyses.

Table S2: Sample information for the 102 Brassica rapa accessions used in this study.

Table S3: Brassica rapa gene IDs for candidate genes identified by our analyses for each crop variety. $\mathrm{TO}=$ toria, $\mathrm{IS}=$ Indian sarson, $\mathrm{PC}=$ pak choi, and CC $=$ Chinese cabbage.

Table S4: Total number of candidate genes identified in our analyses and their distribution on the ten chromosomes of Brassica rapa. 
Table S5: Detailed information of the five Brassica rapa domestication candidate genes identified in all three of our genome scan approaches. Annotation information was obtained from the Brassica Database (brassicadb.org/brad/).

Table S6: Chi-square test results for POInT paleolog enrichment in candidate gene lists and Mann-Whitney $U$ tests of POInT paleolog nucleotide diversity.

Table S7: Summary of the B. rapa candidate genes identified from published mapping studies. Brief description of functions, classical gene names, and Brassica rapa gene IDs are given for each candidate gene. Paleolog status is indicated as Y (yes) or $\mathrm{N}$ (no). If the candidate gene was also identified in our genome scans, the type of scan is indicated with S (SweeD) or D (Differential gene expression). 